\title{
Polymer and heterocyclic compounds their utility and application as drug
}

\begin{abstract}
Polymers and heterocyclic compounds play an important role in pharmacy according to their functions as excipients and inert carriers of other pharmacological active compounds. This review article discuss the recent developments in using this compounds in pharmaceuticals applications as active compounds in both as co drug or drug carriers as well as in delivery systems concerning drug therapy. These polymers and heterocyclic compounds according to their chemical character play important rules in many biological system and that is why we are here discussing there importance in many biological events such as anti microbial, antihyperkalemia and anti cancer agents.
\end{abstract}

Volume 3 Issue 2 - 2019

\author{
Mohammad S Alajely \\ Chemistry department, College of Education, Mosul University \\ Mosul, Iraq
}

Correspondence: Mohammad S Alajely, Chemistry department, College of Education, Mosul University Mosul, Iraq, Emailmohamadalajelee@yahoo.com

Received: January 22, 2019 | Published: March 05, 2019

Keywords: polymer, anticancer drug, polydiacetylenes

\section{Introduction}

\section{Polymer as drug and drug carriers}

It is important that the drug must reaches its site of action at a nearly the same concentration and that its therapeutic dose range have to be remains constant over a long period of time during to its pathway toward the desired target. However, the action of pharmaceutical agents was found to be effected by many factors, including degradation during its pathway, their interaction with other target cells which is not the desired one, and their inability of penetration to the body tissues because of their chemical strecture morphology such as allynments or the functional groups are not well fit to join its target cell. So according to the above stated reasons, many chemical compounds are being studied to achieve a greater pharmacological importance; among the studied compounds are, drug carriers polymers and drug polymers in which them self acts as drug. These polymers are types of systems usually important for time- and distribution-controlled drug delivery. The mechanisms of these polymers used in controlled release process needs polymers having variety of physiological and chemical properties so that to perform its action as dug. Also for hetero cyclic compounds to reach its target have not to be effected and lose any group designed for this purposes. It was known that several types of polymers have been tested as drug delivery systems, including nanoand micro-particles, nano- and micro-spheres, capsosy part of its moleomes, and micelles. ${ }^{1-4}$

In all these systems, drugs can be encapsulated or joints to the polymer backbone. These drug polymer or drug carrier polymers have been used for a verity of treatments such as antineoplastic activity, bacterial infections and inflammatory desires, sequestrate in addition to vaccine. It was also known that many cancer drugs are nearly $90 \%$ heterocyclic compounds. ${ }^{5}$ These compounds were used as anticancer agents due to their being common and widely spread in nature, with numerous number cellular and mechanistic pathways for their interactions with defferents sites at the same time. It is also worth to note that there are variety of metabolic pathways regarding using cellular cancer pathology which can be attributed to heteterocyclic compounds. In this article we are going to introduce the most important heterocyclic and polymeric compounds incorporated to cancer and other therapy in both areas the market and also that which are in development in both of using polymer and heterocyclic chemistry and also discussing their properties that make them valuable as drugs.

\section{Results and discussion}

\section{The slow release of drug using acrylic Itaconic co- polymer}

This study was published elsewere ${ }^{6}$ and showed regular release of anti biotic (in vivo) within a period of 14 days on 4 groups of animals (rabbits) Figure 1. Polymer synthesis and conditions were illustrated below Scheme1. Drug-Polymer associated with Tuberculostatic Activity Based on Poly (N-Vinyl Pyrrolidone-alt-Itaconic Anhydride and Novel Aminoacid Hydrazides was also investigated by Delia G, et al., ${ }^{7}$ The polymer synthetic pathway can be shown as below Scheme $2 \&$ Scheme 3 . This polymer showed significant activities against Tuberculosis as it was stated above.

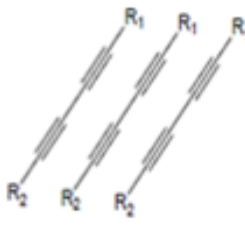

self-assembly
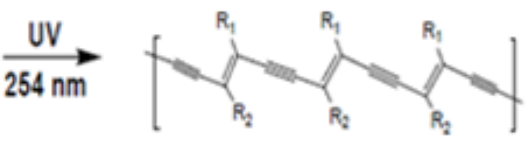

ene-yne alternated polymer

Figure I Polydiacetylene (PDA) synthesis schema via self-assembly polymerization of diacetylene monomers.

\section{Polydiacetylenes as bio sensor}

Usually polydiacetylenes in their strectures act as a by-layer molecules similar to that of the cell wall which will used to diagnose a variety of common diseases, depending on polymer fictionalization with suitable moiety such as sugar group or lipid or others ${ }^{8}$ so it will becomes bio sensor due to collar change phenomena created by the interaction of this group with malarial toxins or any other events. The color change is irreversible so that it can be used to diagnose several diseases the polymerization process can shown as below: For example, GM1 and GT1b gangliosides, which are present on the surface of intestinal cells and at neuromuscular junctions, were utilized for colorbased detection of cholera toxin and botulinum neurotoxin. For this synthesis, $5 \%$ ganglioside lipid was used as a function that be used as a site of action against the targeted site, Using a higher concentration it was found that it will inhibits polymerization of the diacetylene and stops its formation and as are selt no diagnostic ability for this polymer. ${ }^{8}$ Brazilian researchers in October 2010 have synthesized 
10,12 pentacosadyinoioc acid (PCDA) with N-[(2-tetradecan amide)ethyl]) ribonamide (TDER) vesicles to determine the colorimetric response induced by pathogenic bacteria (Staphylococcusaureus and Escherichia coli) (Figure 2).

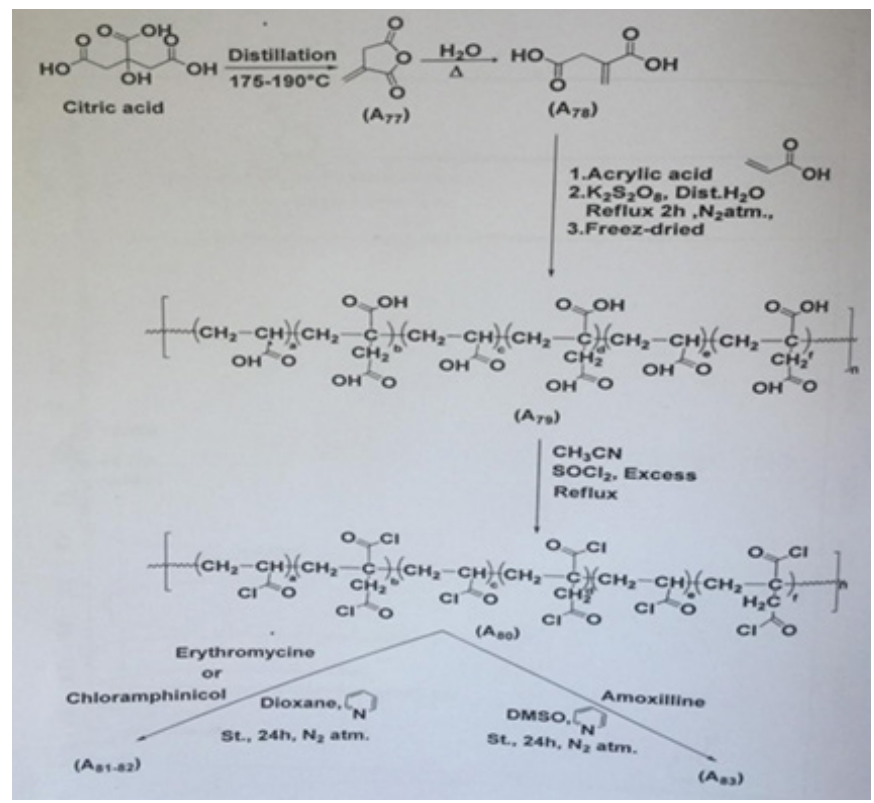

Scheme I Synthesis of Acrylic itaconic co polymer loded with antibiotics.<smiles>[Y10]c1ccc(C(=O)NC(CCCC(N)=O)C(=O)NC(CCC(N)=O)C(=O)OC(C(=O)O)c2ccc([R])cc2)cc1</smiles>

I, IV R= - $\mathrm{NO}_{2} ;$ II, V R= -NH-CHO; III, VI R= -NH-CO-CH

Scheme 2 The formation of amidizolonyl amino acid as aresidue of the polymer.

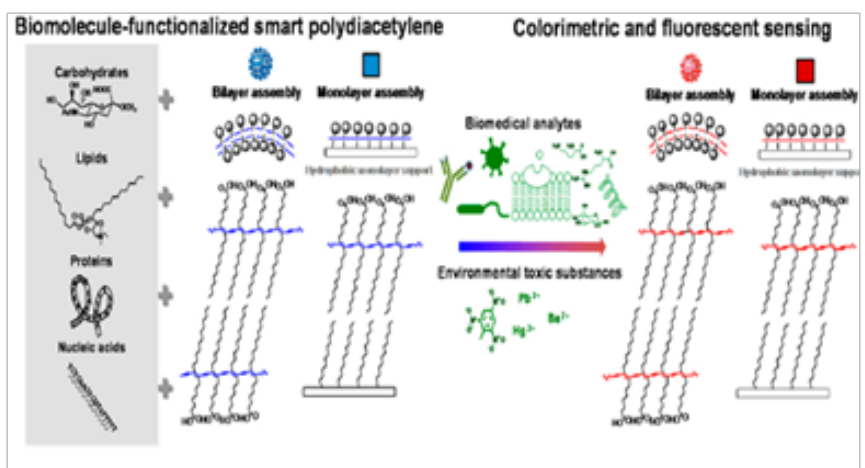

Figure 2 Biomolecule-functionalized Polydiacetylene (PDA) based biomedical and environmental sensing.

The addition of bacterial supernatants caused a colorimetric transition in TDER/PCDA vesicles, even in diluted concentrations, which indicate that a certain chemical interactions occur between the vesicles and the released bacterial toxin compounds. This study was important for food packing and Food born bacteriatypes. ${ }^{9}$ Japanese researchers in march 2016 studied imidazolyl Functionalized diacetylenes. ${ }^{10}$ In their study, They report the first example of polydiacetylenes (PDAs), where the PDA-based system acts as both a sensing probe and killer for bacteria. The contact of imidazolium and imidazole-derived PDA with various bacterial strains including MRSA (methicillin-resistant Staphylococcus aureus) and ESBLEC (extended-spectrum $\beta$-lactamase-producing Escherichia coli) results in a color change from blue-to-red of the solution as well as a rapid disruption of the bacterial membrane, which is followed up by transmission electron microscopy and confocal microscopy. The study of Zeta potential analysis was found to support the successful electrostatic interaction between PDA as a positively charge toward the negatively charged bacterial cell wall. This is an example of the dual activity of polymers which acts as biosensors and at the same time as (Bacterial killers). These dual activity of PDS was shown as low (Figure 3).
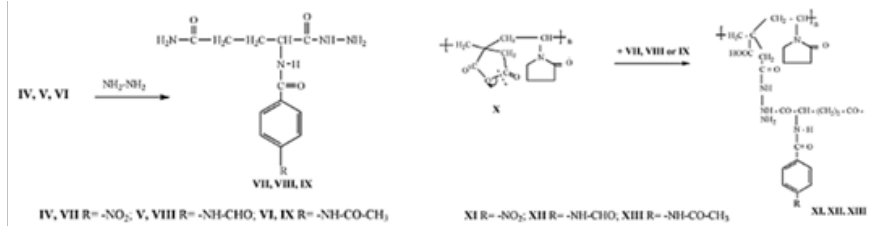

Scheme 3 Showing the steps of preparation of the copoly ( $\mathrm{N}$-vinyl Pyrrolidone-alt-Itaconic Anhydridewith amino acids.

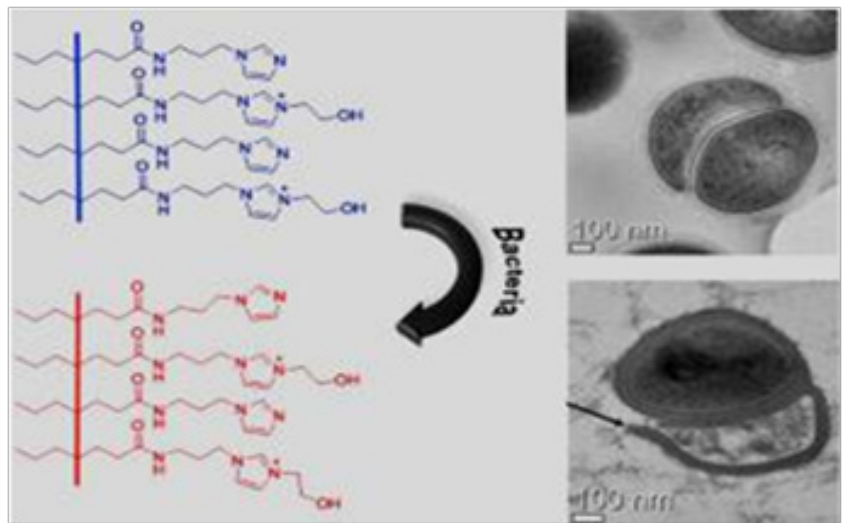

Figure 3 showing the effects of polydiactelyne functionalized with imidazoylgroup on bacteria cell membrane.

\section{Polymers as drugs}

Sodium polystyrene sulfonate (KayexalateVR, SPS) is the first known type of Polymer which is the first synthetic polymers to be widely used as a clinical sequestrate, its strecture is shown in scheme.4 the salt of a polymeric acid, SPS is able to reversibly bind a verity of captions, including potassium. Since potassium is the most popular ion in the colon region as part of intestinal gastric tracts, and is reversibly absorbed in this lower part GI tract, So polymeric agent with capability to such potassium this ion will provide an effective means to reduce serum potassium( Elevated serum potassium)and as a result it will releave kidney diseases .(hyperkalemia) is a disease with incorporated with patient suffering from CKD or cardiovascular conditions which will result in arrhythmia and finally to sudden death[8]. SPS was approved by the FDA for its use on the treatment of hyperkalemia in 1958 SPS which can be synthesized by the polymerization of styrene in in presence of cross linking agent such as divinylbenzene as shown below: Scheme 4 hows the synthetic steps of polystyrene sulfonate 

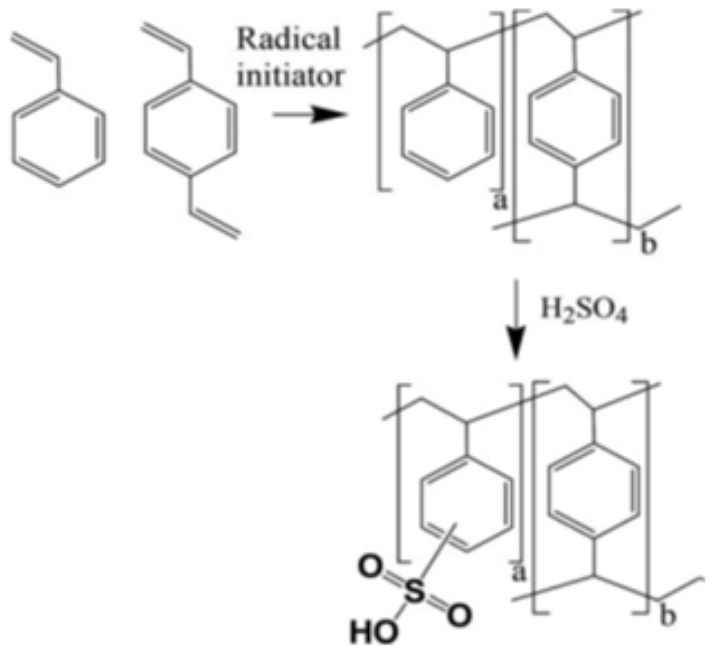

Scheme 4 Hows the synthetic steps of polystyrene sulfonate.

Sevelamer hydrochloride (Renage lVR) was the first polymeric phosphate control and removal of excess phosphate is of highly beneficial to patients with chronic kidney disease CKD where dialysis is unable to maintain safe phosphorus levels. Sevelamer cause to decrease the absorption of dietary phosphorus by binding phosphate in the intestine through ionic interaction with the polyamine polymer. Sevelamer is across linked form of poly (allylamine) which contains primary and secondary aliphatic amine residues and was approved for the treatment of hyperphosphatemia by the FDA in 1998. Thefollowing Scheme 5 shows the synthetic lines for Sevelamer hydrochloride. The synthesis of Sevelamer Hydrochloride as shown above is. Approximately 40 percent of the amine moieties are in the $\mathrm{HCl}$ form, Degree of Cross linking is $10 \%$. The Structure of some commercially available polymer used as sequestrate drugs, were as follows: were sorbitol, which is frequently dosed with SPS as a laxative.
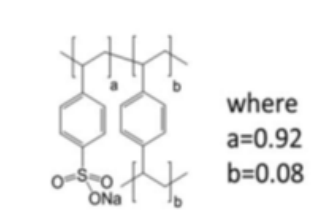

\section{Sodium Polystyrene Sulfonate $\left(\right.$ Kayexalate $^{\bullet}$ )}

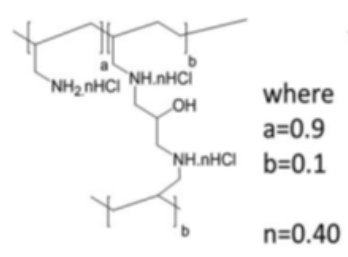

ievelamer hydrochloride (Renagel ${ }^{*}$ )

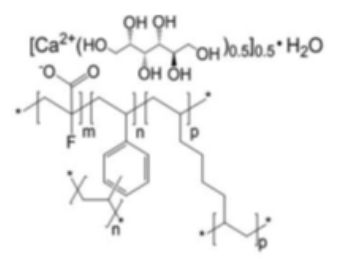

\section{where \\ $\mathrm{m}=0.91$ \\ $n+p=0.09$}

The risk of swelling of the above synthesized drugs Leeds to some improvements to the synthetic pathways stated above so that to increase of its capacity and reducing its swelling property sevelamer is changed into cross liked N,N, N,N-tetrakis (3-aminopropyl) butane-1,4-diamin Scheme 6 and Scheme 7 as illustrated below support the safety profile in clinical studies of up to 52 weeks. This polymer is proved for treatment of hyperphosphatemia by FDA in $1998 .{ }^{10}$ Patiromer is a non-absorbed, potassium-sequestering polymer which is a cross linked form of poly (fluoroacrylic acid). The fluorine substituent cause to lowers the $\mathrm{pKa}$ of the acid group in patiromer compared to acrylic acid such that a higher proportion of acid groups are available for ionic binding. Suspension polymerization during patiromer manufacture allows for the formation of monodisperse with uniform polymer particles, spherical shape, having controlled size distribution, and low swelling. The bead particles have a diameter of around $100 \mu \mathrm{m}$. Patiromer was approved by the FDA for the treatment of hyperkalemia in 2015 based on clinical studies which revealed effective potassium lowering and acceptable safety profile in clinical studies of up to 52 weeks duration. The synthetic pathway for this cross linked polymer is shown below: Scheme 7-Synthetic steps of Patiromer.

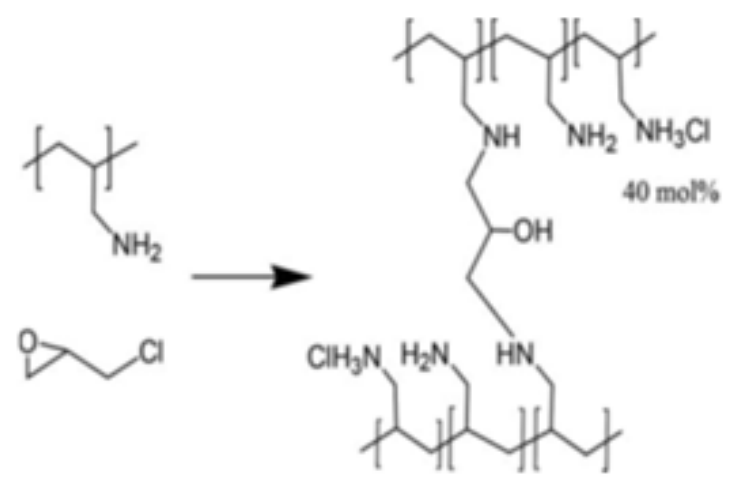

Scheme 5 Represent the formation of polyallyl amine as a resedue of sevelamer strecture.<smiles>NCCN(CCN)CCCN(CCN)CCN</smiles>

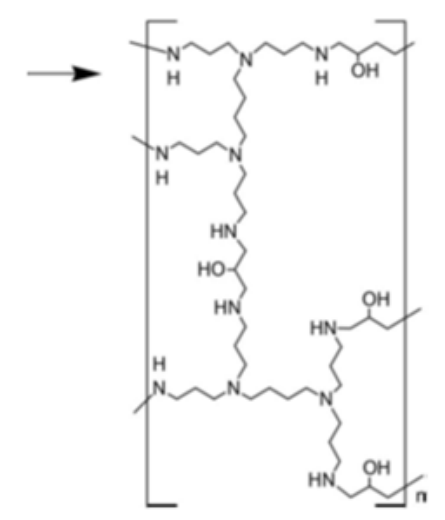

Scheme 6 Showing the network formation of patiromer amine residue.

\section{Heterocyclic compounds in anti-cancer drug design strategy}

As it was stated in the introduction part of this review that heterocyclic compounds are widely spread in nature they have become so important for anti-cancer drug design and other therapeutic path 
ways. They represent a precursor and building molecules of a variety of drugs or co drug compounds depending on the active functional groups they have, Depending on the above facts these heterocyclic compounds becomes the bases of most drugs designing systems. The therapeutic time and ageing of drugs As well as enzymatic binding all are associated with heterocyclic moieties, So heterocycles are becomes good choice when designing molecules that will interact with targets and disrupt the biological pathways associated with cancer progression Especially pathways related to cell growth and development are often targeted by such as anti-cancer therapies as stated above . Moreover there are versatile method by which can modified the stricture of heterocyclic rings with any additional group or element which finally willed allows them to cover a broad area of chemical interaction sites, and as a result will quilting them as excellent starting points for cancer therapeutic designing and developments. Finally and as a result of the stated above facts, Heterocyclic compounds play an important rules in drug designing and development and provides the market with a lot of new pharmaceutical compounds every year for both cancer and other therapeutic drugs.
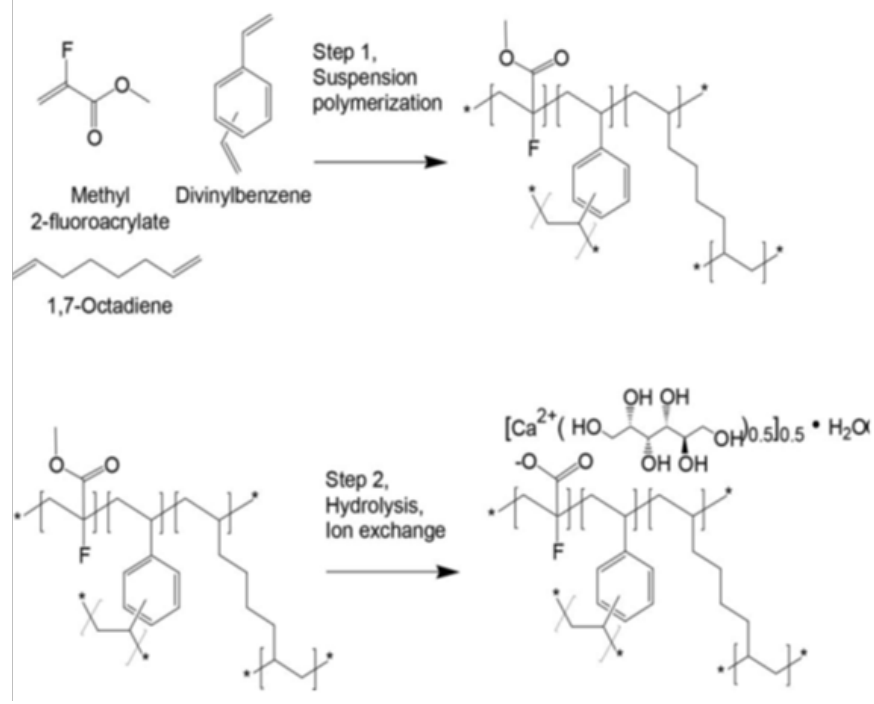

Scheme 7 Synthetic steps of Patiromer.

It is worth to say that nearly $65 \%$ of the anti-cancer drugs granted market approved by FDA bet ween2010-2015 form the basis of many of the anti-cancer agent currently in development today are Nitrogenbased heterocycles. ${ }^{11}$ It was approved by the FDA between 2010 and 2015 that nitrogen based heterocyclic compounds forms two third of the commercial cancer drugs. Nnitrogen heterocycles, in doles are among the most valuable, in research demonstrated their ability to induce cell death in a number of cancer cell lines2 During the last few decades, indole and its derivatives have been shown to have biological pathways against cancer through the on growing research and developments. These include the prevention of cell signaling normal cell cycle progression, tumor vascularisation and DNA repair, as well as the ability to induce cellular oxidative stress and cell death The most important was an early used commercially indole-based anticancer agents are vincristine and vinblastine which are recognized for their tubulin polymerization inhibition since the early-mid 1960s and both still of clinical importance today. Vincristine (Figure 4) is used for the treatment of acute lymphoblastic leukaemia and both Hodgkin's and- non-Hodgkin's lymphoma. ${ }^{12}$

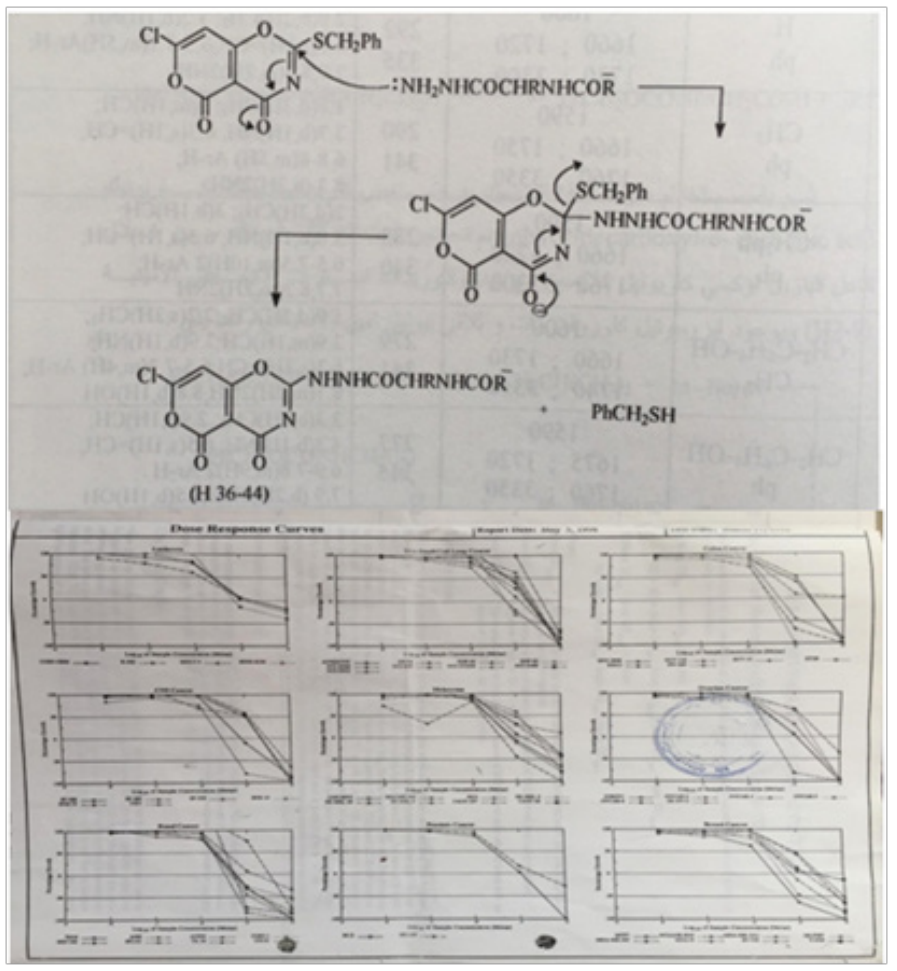

Scheme 8 Shows the synthetic steps of amino chloropyrano oxazine compounds and the graphical results showing the effects of these compounds on different types of cancer cells.

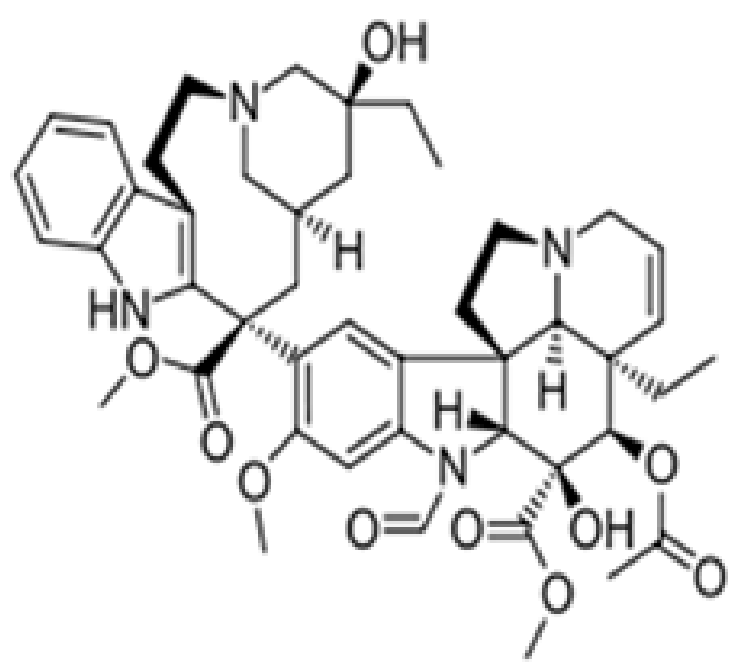

Figure 4 Vincristine is used for the treatment of acute lymphoblastic leukaemia and both Hodgkin's and- non-Hodgkin's lymphoma.

The development in heterocyclic compounds its utility and applications toward cancer screening studies showed a lot of works in this area of drug discovery as an examples coumarin and oxazine compounds. For coumarin containing heterocylles ${ }^{13}$ the following compounds showed antitumor activities against two cell lines breast carcinoma Michigan Cancer Foundation-7 (MCF-7) and hepatocellular carcinomarin (HepG-2), at the National Cancer Institute, Cairo, Egypt using 5-fluorouracil as standard drug. The strectureot the synthesized compounds were illustrated below. While for, oxygen 
and nitrogen containing heterocycle (Chloro pyrano Oxazines) compounds and according to the following Scheme 8 together with the graphical curves(NCI report) showed remarkable results for these types of compounds. Scheme 8 shows the synthetic steps of Amino chloropyrano oxazine compounds and the graphical results showing the effects of these compounds on different types of cancer cells The above investigation revealed that the studied compounds kill about 89 $-100 \%$ cancer cell (6-8 types) at the same time. ${ }^{14}$
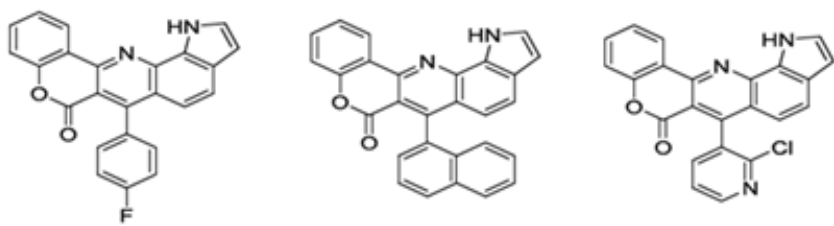

Figure 5 Dericoumarin derivatives as anti tumor agents.

\section{Acknowledgments}

None.

\section{Conflicts of interest}

The author declares there is no conflict of interest.

\section{References}

1. Hyung-Kwan Chun, Chong CS, Chong Su Cho. Mucoadhesive drug carrier based on interpolymer complex of poly (vinyl pyrolidone) and poly(Acrilic acid)prepared by template polymerization. J Control Release. 2002;17;81(3):327-334.

2. Vllalobos, Patricio, Charez, et al. The application of polymerized lipid vesicles as colometric biosensors for real-time detecions patthogens in drinking water. Electronic J of Biotechnology. 2001;15(1):1-5.

3. Kristy S, Mcketrin, Alexandra A, et al. Biosensors and nanobiosensors for therapeutic drug and response monitoring. 2016;2:16.
4. Suh, Lis, Sin Y, et al. Nanomaterial-based biosensors for biological dections. Advanced Health care Technologies. 2012;3:19-29.

5. Nayyert, Mohammadian, Karimz, et al. S-NICS investigation for heterocyclic antic anser compounds. Orient $J$ of chemistry. 2017;33:1595-1602.

6. Mohammed S Al-Ajely, AY Qasim. Synthesis of some streoregular polydiacetylenes and the slow release of drug using some linear polymers. Pakistan J of chemistry. 2012;2(3):142.

7. Delia G, Mihaela N, Catalina L, et al. Drug-polymer conjugate with tuberglostatic Activity, Based on poly (N-Vinyl Pyrrolidone-alt-Itaconic Anhydride) and Novel Amino acid Hydrazides. Polymer-Plastics Technology and Engineering. 2013;52(12):1213-1219.

8. Eunae Cho, Seunho Jung. Biomolecules-Functionalized smart polydiacetylene for biomedial and environmental sensing. Molecules. 2018;23(1):107.

9. Ana Clarissa S, Pires Nilda de Fátima Ferreira, Soares Luis Henrique Mendes da Silva. Colorimetric response induced by pathogenic bacteria, Sensors and Actuators B: Chemical ELSEVIER. 2010.

10. Lee S, Cheng H, Chi M, et al. Sensing and antibacterial activity of imidazolium-based conjugated polydiacetylenes. Biosensors and Bioelectronic. 2016;77(15):1010-1019.

11. Martins P, Jesus J, Santos S, et al. Heterocyclic Anticancer compounds: Recent advances and the paradigm shift toward the use of nanomedicine toolbox. Molecules. 2015;20(9):1689-1691.

12. Morsy SA, Farahat AA, Nasr MNA, et al. Synthesis, molecular modeling and anticancer activity of new coumarin containing compounds. Saudi Pharm J. 2017;25(6):873-883.

13. Abbas Al-Mulla. A Review: Biological Importance of Heterocyclic Compounds. Der Pharma Chemica. 2013; 9(13):141-147.

14. Mohammad S Al-Ajely, FThanoon Al-Abachi. Iraqi patent No. 2807. 2008 . 